\title{
Diagnóstico del trabajo colaborativo en un centro escolar como indicador de inclusión educativa
}

\author{
A School Diagnosis About Collaborative Work as an \\ Indicator of Educational Inclusion
}

\begin{abstract}
Diagnóstico do trabalho colaborativo em uma escola como indicador de
\end{abstract} inclusão educacional

María Fernanda Bonilla Serrato

Universidad Juárez del Estado de Durango, Durango, México

ferbonilla0@gmail.com https://orcid.org/0000-0002-6065-2508

Jaime Fernández Escárzaga*

Universidad Juárez del Estado de Durango, Durango, México

jaimefer14@hotmail.com https://orcid.org/0000-0002-8897-5851

Marco Antonio Vázquez Soto Universidad Juárez del Estado de Durango, Durango, México marco0709@hotmail.com https://orcid.org/0000-0002-8449-1120

*Autor de correspondencia 


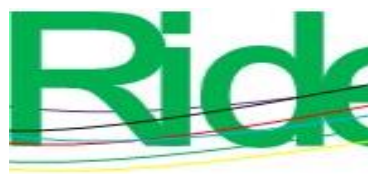

Revista Iberoamericana para la Investigación y el Desarrollo Educativo ISSN 2007 - 7467

\section{Resumen}

La inclusión educativa es una filosofía de intervención que orienta la atención de la comunidad de un centro escolar hacia la diversidad de todos sus alumnos. En México el sistema educativo está obligado a desarrollar en las aulas prácticas inclusivas. Sin embargo, las acciones que se realizan generalmente se reducen a la integración de los alumnos con discapacidades. Este manejo discrecional de la inclusión educativa es lo que motivó a desarrollar un diagnóstico en una institución de la periferia de la ciudad de Durango, Dgo., México. El objetivo del diagnóstico fue encontrar cuáles eran las prácticas inclusivas que se llevaban a cabo en la escuela respecto al trabajo colaborativo, con la finalidad de realizar posteriormente un proceso de intervención que contribuyera a fortalecer los hallazgos positivos y mejorar las áreas donde se tuvieran pocos avances.

El método para realizar el diagnóstico fue cualitativo de tipo observacional fenomenológico. Se rescataron los datos empíricos empleando la técnica de observación no participante y la entrevista en profundidad. Este proceso tuvo la duración de un mes, con una asistencia diaria a la primaria dentro del horario escolar. Durante el diagnóstico, la intervención y el análisis de los resultados, el material recopilado se organizó en diez categorías. En este artículo se presentan solamente las categorías que corresponden al resultado del diagnóstico. La información obtenida fue contrastada con fuentes teóricas que sustentaban y explicaban los hallazgos.

Los resultados encontrados muestran que la escuela no tiene elementos para considerarse como centro inclusivo. La comunicación del director hacia los docentes es deficiente; su falta de asertividad ha provocado desconfianza, ansiedad y resentimiento. Su liderazgo, lejos de fortalecer el trabajo colaborativo, ha generado incomodidad en los integrantes del cuerpo académico. El diagnóstico realizado permitió identificar los elementos para desarrollar un proceso de intervención orientado desde la psicología educativa con un esquema de investigación-acción.

Palabras clave: inclusión, intervención educativa, psicología educativa. 


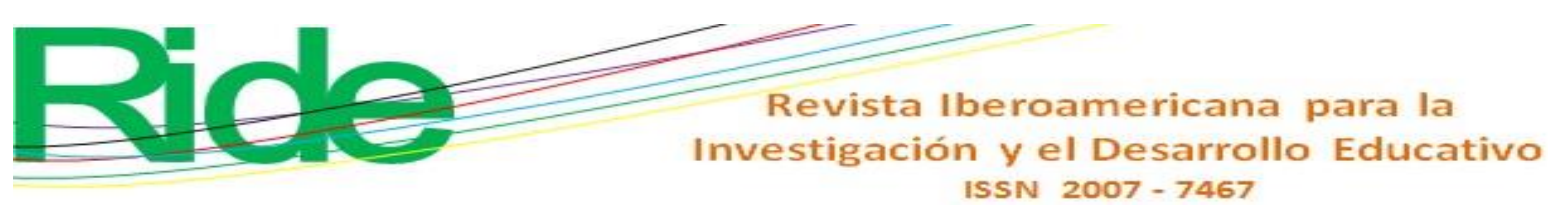

\section{Abstract}

The educational inclusion is an intervention philosophy that orients the school community to achieve the educational attention in all the student's diversity. In Mexico, because of the international agreements that it's been subscribed, the educational system is obligated to develop inclusive practices in all its schools. However, the actual actions are generally reduced to the integration of students with some kind of disability in the classrooms. This discretional use of educational inclusion is what motivated to make a diagnosis in a school located in the outer fringe in the city of Durango, Durango, Mexico. The objective of the diagnosis was to find out the inclusive practices that the school makes in relation to collaborative work, with the purpose of implement subsequently an intervention process that will contribute to strengthen the positive findings and areas for improvement in the school.

The diagnosis was made with a qualitative method using a phenomenological observational type. The data were rescued using the non-participant observation technique and the depth interview. This process lasted a month, with daily attendance at the primary school hours. The material was organized in ten categories that were used in the diagnosis, intervention and the analysis of the results. In this article only the categories that correspond to the result of the diagnosis are presented. The information obtained was contrasted with the theoretical sources that supported and explained the findings, throwing as conclusions, the situation in which the school is located.

The results show that the school doesn't have enough elements to be consider as an inclusive institution. The communication is not efficient between the principal and the teachers; the lack of assertiveness in the principal has caused anxiety, mistrust and resentment. His type of leadership has led to create discomfort among the teachers weakening the collaborative work. The collaborative work is non-existent among teachers, students and parents because of the principal's restrictions for the parent's access into the school. The diagnosis provided the elements to develop an intervention process guided by the educational psychology using an action-research orientation.

Keywords: inclusion, educative intervention, educational psychology. 


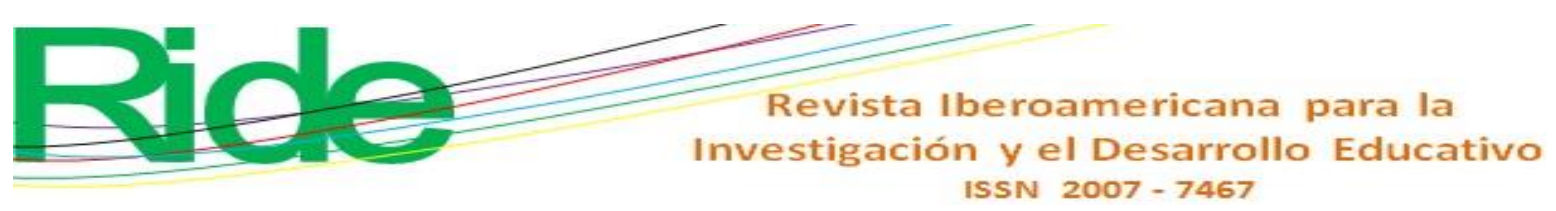

\section{Resumo}

A inclusão educacional é uma filosofia de intervenção que orienta a atenção da comunidade de uma escola para a diversidade de todos os seus alunos. No México, o sistema educacional é obrigado a desenvolver aulas práticas inclusivas na sala de aula. No entanto, as ações realizadas são geralmente reduzidas à integração de alunos com deficiência. Esta gestão discricionária da inclusão educacional é o que motivou o desenvolvimento de um diagnóstico em uma instituição na periferia da cidade de Durango, Dgo., México. O objetivo do diagnóstico foi descobrir quais foram as práticas inclusivas que foram realizadas na escola em relação ao trabalho colaborativo, com o objetivo de posteriormente realizar um processo de intervenção que contribuiria para fortalecer os achados positivos e melhorar as áreas onde houve pouco progresso. .

O método para o diagnóstico foi qualitativo do tipo observacional fenomenológico. Os dados empíricos foram resgatados usando a técnica de observação não participante e entrevista em profundidade. Este processo durou um mês, com uma frequência diária na escola primária durante o horário escolar. Durante o diagnóstico, intervenção e análise dos resultados, o material coletado foi organizado em dez categorias. Este artigo apresenta apenas as categorias que correspondem ao resultado do diagnóstico. As informações obtidas foram contrastadas com fontes teóricas que apoiaram e explicaram os achados.

Os resultados encontrados mostram que a escola não possui elementos para ser considerada como um centro inclusivo. A comunicação do diretor com os professores é ruim; sua falta de assertividade causou desconfiança, ansiedade e ressentimento. Sua liderança, longe de fortalecer o trabalho colaborativo, gerou desconforto nos membros do corpo acadêmico. $\mathrm{O}$ diagnóstico permitiu identificar os elementos para desenvolver um processo de intervenção orientado pela psicologia educacional com um esquema de pesquisa-ação.

Palavras-chave: inclusão, intervenção educativa, psicologia educacional.

Fecha Recepción: Abril 2019

Fecha Aceptación: Agosto 2019 


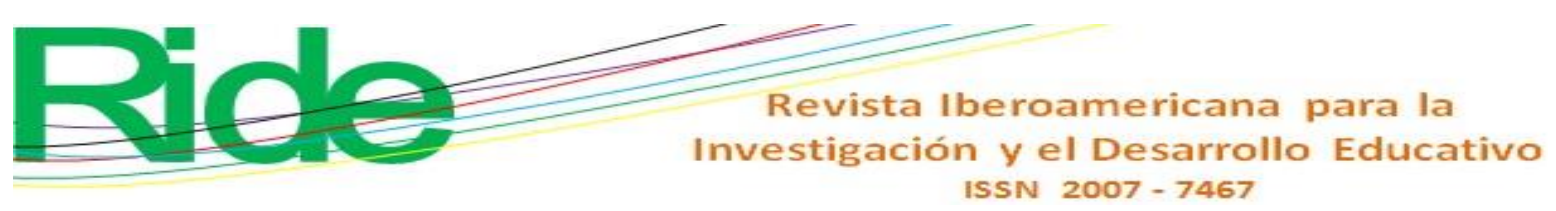

\section{Introducción}

El presente artículo es el reporte de la primera fase de la investigación y comprende el diagnóstico que se hace en un centro educativo de la periferia de la ciudad de Durango, Dgo., México. Las otras fases de la investigación fueron la intervención y finalmente la evaluación posintervención, a fin de valorar la eficacia de esta. Es un trabajo de investigación-acción: el investigador es el protagonista del proceso de transformación.

En esta sección se reporta el diagnóstico que se realizó con el objetivo de establecer el proceso de intervención en la comunidad escolar, y se tomó como referente inicial el trabajo colaborativo que se lleva a cabo dentro del plantel, ya que es un indicador importante en la inclusión educativa. Para la educación inclusiva es necesario que los sistemas educativos se orienten hacia la participación y el compromiso de toda la comunidad escolar para generar un cambio intrínseco de la política, la cultura y la práctica educativa; y crear un nuevo ambiente donde se considere la diversidad como una oportunidad para aprender y también para enseñar con ella, garantizando el acceso, la permanencia, la participación y el progreso de todos los estudiantes (Porter y Towell, 2017; Escobar, Albores y Guillén, 2017).

La importancia que este primer momento de diagnóstico posee es que, a diferencia de otros países, en México la educación inclusiva es una filosofía de educación que no está arraigada en los docentes, ni en los directivos, ni en las políticas educativas del Gobierno mexicano. A pesar de que el Departamento de Educación Especial de la Secretaría de Educación Pública (SEP) pregona la adopción de la educación inclusiva en un gran número de planteles, la realidad es que las prácticas que realiza el personal de esa área siguen manteniendo la vieja escuela de la educación especial y de los grupos integrados.

En México son pocos los proyectos educativos inspirados por los principios de la educación inclusiva. La filosofía de la educación para todos se encuentra escasamente concretada en algunas actividades "inclusivas" que se realizan de forma aislada.

Aunque se sabe que la responsabilidad del sistema educativo es proporcionar oportunidades de aprendizaje de calidad para todos los niños y niñas (San Martín, Villalobos, Muñoz y Wyman, 2017), se continúan desarrollando barreras para el aprendizaje; barreras que existen por emplear un currículo mal diseñado, maestros poco o mal capacitados, con medios de instrucción inapropiados y edificaciones inaccesibles para los alumnos (Organización de las Naciones Unidas para la Educación, la Ciencia y la Cultura [Unesco], 


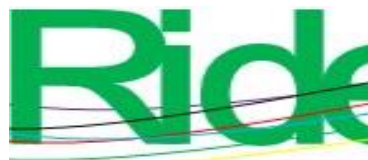

Revista Iberoamericana para la
Investigación y el Desarrollo Educativo
ISSN $2007-7467$

acción de tipo transversal con un enfoque cualitativo durante el periodo de un año en la institución educativa del corregimiento de Yarima, Colombia, con el objetivo de formular e implementar una propuesta para el mejoramiento de la calidad educativa de la escuela siguiendo los principios inclusivos.

Los resultados obtenidos muestran el gran potencial que las comunidades de aprendizaje pueden alcanzar cuando adoptan una estrategia de educación inclusiva, ya que permitieron sacar provecho de las opiniones de estudiantes, profesores, padres de familia y demás pobladores en torno a la educación, y enriquecieron y permitieron llevar a cabo cada una de las iniciativas propuestas. Hubo un incremento en las puntuaciones de las evaluaciones a nivel nacional; se fortaleció el vínculo entre los padres de familia con el proceso educativo de sus hijos; del mismo modo, se desarrolló una identidad comunitaria entre alumnos, docentes y el entorno local; también se sensibilizó al docente sobre su función en el proceso de enseñanza a través de la innovación de sus prácticas y la capacitación continua (Beltrán et al., 2015).

Mientras tanto, un estudio realizado en México por Flores, García y Romero (2017) buscó identificar las prácticas inclusivas de docentes que forman parte de una escuela normalista, con la intención de localizar los cambios estructurales en el sistema educativo que han provocado descontento y confusión en profesores que intentan desarrollar su enseñanza a partir de principios inclusivos. Los resultados obtenidos indican que se requieren mayores apoyos en las condiciones físicas del aula, en la metodología de enseñanza y en el establecimiento de la relación maestro-alumno; esto como parte la necesidad de un plan de actualización docente durante la formación de este profesionista.

La inclusión educativa implica la participación y colaboración de toda la comunidad escolar, incluyendo a los directivos, a los profesores, a los alumnos y a los padres de familia. El compromiso de cada uno de ellos y la aportación que realicen durante este proceso asegura que la transformación de las prácticas educativas permita a su vez el cambio cultural de la comunidad hacia los principios de equidad e igualdad para fortalecer una pedagogía inclusiva que desarrolle y fortalezca la enseñanza y el aprendizaje. Esto al contemplar que la diversidad da la oportunidad para aprender y enseñar con ella, y garantiza el acceso, la permanencia, la participación y el progreso de todos los estudiantes (Porter y Towell, 2017; Escobar et al., 2017). 


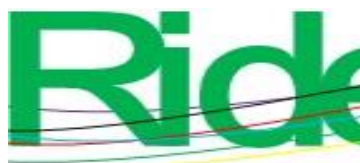

Revista Iberoamericana para la Investigación y el Desarrollo Educativo ISSN $2007-7467$

No obstante, formar un centro educativo en donde sus miembros se desarrollen con un compromiso hacia la inclusión implica que en sus prácticas se demuestre la existencia de una filosofía de democracia e igualdad; en donde la calidad educativa es el resultado de la organización, la planificación y el desarrollo de estrategias en colectivo en la toma de decisiones que den respuesta a las necesidades educativas y que generen redes de apoyo durante el trabajo en colaboración. Esto con el fin de mantener flexibilidad en las estrategias y planeaciones para la adaptación del currículum respecto a las necesidades de los alumnos (Moliner, 2013; López, 2011).

Cada miembro cumple funciones específicas que acercan o alejan al centro escolar de su proceso inclusivo. Ante el desafío de acercar a todos sus estudiantes a los conocimientos académicos, el docente requiere de servicios de asesoramiento y supervisión psicopedagógica (Echeita, 2013) por parte de especialistas y directivos del centro. Esto como consecuencia de que en el proceso de enseñanza-aprendizaje, en muchas ocasiones, se evidencian deficiencias pedagógicas del profesor y son estas las que impiden que este proceso sea exitoso para todos los alumnos (García, García y Reyes, 2014), lo que genera en el docente una actitud neutral o negativa hacia la inclusión (Granada, Pomés, y Sanhueza, 2013). Por lo que se requiere que el profesorado disponga de buenas prácticas educativas que atiendan las necesidades del grupo.

Otra de las figuras necesarias en este proceso inclusivo es la de los directivos: son guías y líderes del centro escolar. Ellos, a través de su interacción, potencian e impulsan una cultura inclusiva (Fernández y Hernández, 2013). Su función consiste en definir los objetivos, establecer las normas de trabajo y los lineamientos a seguir (Sanabria y Castañeda, 2016), pero también implica comunicar a sus profesores lo que se espera de ellos durante la puesta en marcha de las actividades. La comunicación que él establezca debe favorecer el desarrollo de todo el personal del centro, y debe procurar tomar en consideración las expectativas de los profesores, sus necesidades, preocupaciones y experiencias. De esta manera, se fomenta un ambiente de confianza y participación que posibilita la autonomía y organización del cuerpo docente en un marco de trabajo compartido (Fernández y Hernández, 2013).

Los docentes, al igual que el resto de la comunidad escolar, deben generar un sentido de comunidad basado en la satisfacción en el trabajo, la motivación y la identidad hacia el centro. Esto requiere de la vinculación colectiva de los integrantes del equipo. Es decir: las relaciones afectivas que establezcan los profesores permitirán que la práctica educativa se 


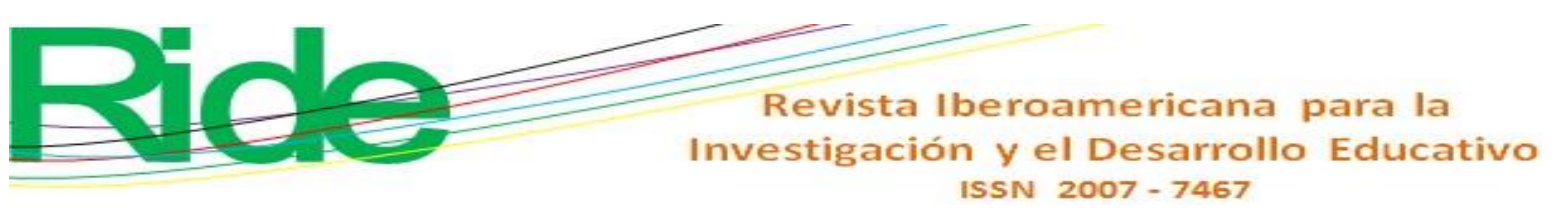

desarrolle desde la reflexión conjunta y no de forma individual al interior de su aula; al mismo tiempo que incrementarán el compromiso y responsabilidad del docente por la educación de los alumnos: aportarán a su desarrollo profesional, generarán identidad y la noción de que el cuerpo profesional trabaja en colaboración (Bolívar, 2013; Matus, 2013).

Sin embargo, en algunos centros la implementación de equipos de trabajo colaborativo no se considera una tarea fácil. Muchas veces debido a una falta de compromiso en la educación de los alumnos, acompañado por el deseo de obtener resultados rápidos y la costumbre de tomar decisiones a partir de la conveniencia individual; la débil lealtad entre los miembros y el poco involucramiento en las relaciones interpersonales hacen que el trabajo se realice con inestabilidad e incertidumbre (Sisto, 2009). El compromiso con el trabajo se producirá solo cuando se ofrezcan vínculos de confianza, que crecen y se fortalecen al manifestar aprecio hacia el otro, y al ser capaces de reconocer y estimar el mérito de alguien (García, 2013). Trabajar en colaboración implica que cada uno de los individuos tendrá bajo su responsabilidad cierta tarea que se le ha confiado y que, por sus cualidades individuales, se asegura el éxito de esta.

\section{Materiales y métodos}

El reporte de la investigación que hoy se presenta es la primera fase de un trabajo de intervención, en donde se buscó disponer de un diagnóstico que permitiera definir con claridad las oportunidades de una intervención desde la psicología educativa en un centro escolar en particular con la finalidad de mejorar los indicadores de educación inclusiva. Para ello se diseñó una estrategia de observación no participante durante un mes. Esta observación se hizo durante el horario de la jornada laboral completa, y se realizaron entrevistas a profundidad con los informantes clave. Las observaciones se registraron en una bitácora y se hicieron diarios de campo; las entrevistas fueron grabadas en audio y transcritas posteriormente para su análisis.

Para el análisis de la información obtenida se establecieron diez categorías y seis subcategorías, que fueron revisadas ampliamente y procesadas utilizando el programa de análisis cualitativo Atlas.ti. En el presente artículo solamente se toman en consideración tres categorías y tres subcategorías, tal y como puede observarse en la tabla 1. 


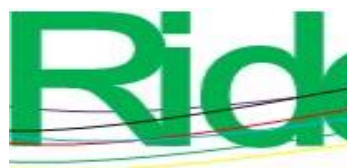

El estudio es de tipo cualitativo, descriptivo y fenomenológico. Los materiales empleados fueron hojas de registro, grabadora, computadora y el programa asistido por computadora mencionado.

En los resultados que se presentan, las referencias textuales de los informantes clave se indican entre paréntesis el párrafo en donde se encuentra la cita en cuestión, según la bitácora elaborada.

Tabla 1. Categorías de análisis con mayor incidencia en el centro escolar

\begin{tabular}{|l|l|}
\hline Categorías & Subcategorías \\
\hline Formas de convivencia & $\begin{array}{l}\text { Estilos de comunicación } \\
\text { Participación entre individuos } \\
\text { Encuentros entre los individuos }\end{array}$ \\
\hline Preferencias del Director con el personal & \\
\cline { 1 - 1 } Evidencia de falta de trabajo colaborativo & \\
\hline
\end{tabular}

Fuente. Elaboración propia

En la tabla 2 se muestra la definición que se dio a cada una de las categorías aquí analizadas. La categoría Formas de convivencia tiene tres subcategorías: Estilos de comunicación, Participación entre individuos y Encuentros entre personas. Además, se incluyen las categorías Preferencias del director con el personal y, finalmente, Evidencias de falta de trabajo colaborativo. 


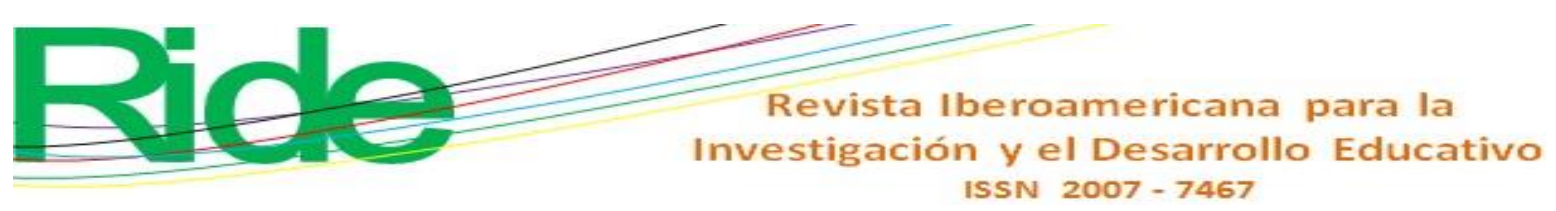

Tabla 2. Definición de categorías

\begin{tabular}{|l|l|}
\hline Categoría: formas de convivencia \\
\hline $\begin{array}{l}\text { Subcategoría: Estilos de } \\
\text { comunicación }\end{array}$ & $\begin{array}{l}\text { Se presenta como las formas en que el líder se expresa } \\
\text { ante las situaciones de la vida escolar. Cómo dialoga, } \\
\text { cómo demuestra su aprecio y cómo relaciona con los } \\
\text { demás. }\end{array}$ \\
\hline $\begin{array}{l}\text { Subcategoría: Participación } \\
\text { entre individuos }\end{array}$ & $\begin{array}{l}\text { Se considera la frecuencia de participación, y si las } \\
\text { participaciones se relacionan con el tema de diálogo y } \\
\text { cómo son tomadas en cuenta por los demás. Crean un } \\
\text { ambiente de posibilidad para actuar, intervenir u } \\
\text { opinar durante las negociaciones que realizan, así } \\
\text { como la voluntad grupal de establecer puntos de } \\
\text { acuerdo. Implica el grado de responsabilidad y } \\
\text { compromiso de sus miembros, aunado al respeto que } \\
\text { se brindan, se protegen y promueven su participación. }\end{array}$ \\
\hline $\begin{array}{l}\text { Subcategoría: Encuentros entre } \\
\text { los individuos }\end{array}$ & $\begin{array}{l}\text { Espacios y tiempos destinados a promover la } \\
\text { convivencia entre los individuos. Prestaciones de los } \\
\text { servicios educativos para facilitar los encuentros. }\end{array}$ \\
\hline $\begin{array}{l}\text { Categoría: Preferencias del director con el personal } \\
\text { Conductas y actitudes que diferencian la relación del director con el personal docente a su } \\
\text { cargo. Cómo coordina y organiza a su equipo; asimismo, cómo los motiva y les demuestra } \\
\text { su afecto. }\end{array}$ \\
\hline $\begin{array}{l}\text { Categoría: Evidencia de falta de trabajo colaborativo } \\
\text { Reconocer y aprovechar las habilidades de cada miembro para desarrollar un objetivo en } \\
\text { común. Demostraciones de apoyo hacia los demás. Ayudas que se ofrecen hacia el otro, } \\
\text { cumplimiento de su labor y constancia de la eficacia de su trabajo. Objetivos propuestos en } \\
\text { común, así como los recursos y medios para realizarlos. Satisfacción a las necesidades del } \\
\text { grupo. El rol del liderazgo. }\end{array}$ \\
\hline
\end{tabular}

Fuente. Elaboración propia

\section{Resultados}

Entre los principales resultados que se obtuvieron en la categoría Formas de convivencia, destaca la manera de comunicación del grupo directivo hacia los docentes, que es descrita, en voz de una profesora, como "de malos modos, tiene sus ratos en que no puedes decirle nada porque te va a tratar bien mal después, normalmente así nos trata a mí y a la maestra E" (párrs. 1789-1790). Cuando se sabe que fomentar un ambiente de confianza y participación, requiere comunicar y considerar las expectativas de los profesores, sus 


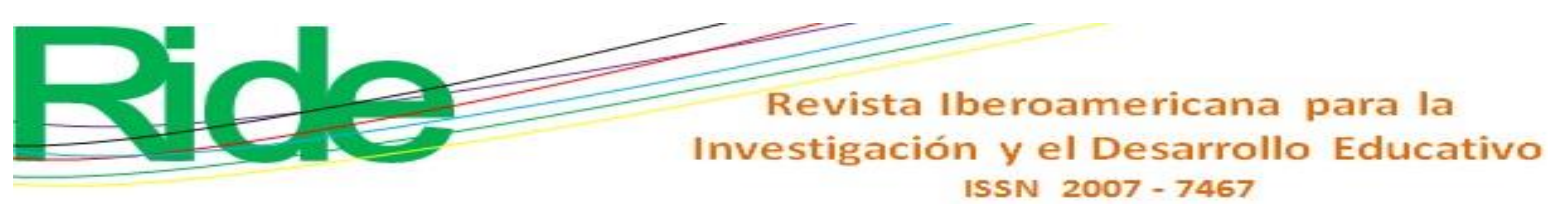

necesidades y preocupaciones (Fernández y Hernández, 2013), la misma profesora comparte lo siguiente:

En los consejos técnicos a veces ni participo porque el director es muy grosero y empieza con sus albures. Es una persona muy preparada... pero por cómo habla ¡no! A mí se me hace muy grosero, hay otros profes que sí le siguen la corriente, pero yo no, a veces hasta me salgo (párrs. 1042-1046).

La figura directiva sirve como guía y líder del centro escolar porque su interacción con otros miembros fortalece el trabajo que se realiza (Barrios, Castillo, Fajardo, Rojas y Nova, 2004). Pero se percibe incomodidad por otra integrante del cuerpo académico, quien comparte que "yo no sé por qué la maestra E se deja de los modos del dire. Yo también me dejaba, pero ya le puse su alto y a mí ya no me trata mal, pero antes sí” (párrs. 2199-2203). Y concluye: "Yo siento que falta un poquito que él a veces sí modere su forma de decirnos algunas cosas" (párrs. 30-31).

En la subcategoría de Participación y encuentros entre los miembros se esperaba que los docentes y los alumnos pudieran realizar trabajo colaborativo con los padres de familia, ya que la participación y colaboración de toda la comunidad escolar asegura la transformación de las prácticas educativas (Porter y Towell, 2017). Los centros escolares requieren de la participación de los padres de familia; sin embargo, una maestra de la escuela que se analiza menciona que "no me gusta pelearme con las mamás porque son más groseras y empiezan a hacer chismes de uno (...), por eso le digo al director para que él se encargue de las mamás y que lo dejen trabajar a uno” (párrs. 2097-2101).

La perspectiva de la institución escolar se hace manifiesta en voz de algunas de las docentes que menciona que el director recomienda que "no permitan que las mamás entren. Si alguna mamá entra por alguna duda de tarea o algo, él va y nos dice: por favor, no, señora, tiene que estar afuera" (párrs. 37-40). Otra profesora justifica que los encuentros con los padres de familia han sido a razón de lo puesto a continuación:

[He] escuchado de comentarios tanto del director como de los profes que ya tienen aquí tiempo, que entre menos contacto tengas con los padres mejor porque te evitas problemas, o sea, lo que es relacionado con los niños, que las calificaciones, que el trabajo, que no trae tarea y ya, pero así como que permitirles un poquito más, que no, que porque luego ya no te los quitas de encima (párrs. 846-851). 


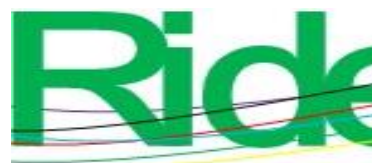

Revista Iberoamericana para la
Investigación y el Desarrollo Educativo
ISSN $2007-7467$

Otro requisito del trabajo colaborativo es la participación entre docentes, ya que la calidad educativa del centro es el resultado de la organización, la planificación y el desarrollo de estrategias en colectivo basado en redes de apoyo durante las tareas (Moliner, 2013). Un profesor comparte:

Nos tenemos que preparar para hacerle las mentadas adecuaciones al niño y uno va para que le ayuden a saber cómo le hago y lo único que nos dice: "pos hágale adecuaciones”; “pos ya las hice ¿ahora qué?”. Y es mi problema porque yo tengo al niño.

La respuesta del director, por su parte, fue la siguiente: "Pues sí, profesor, el que tiene que investigar cómo hacerle es usted” (párrs. 1533-1539); y agregó que lo importante es preguntarse “¿cómo sacar adelante a aquellos alumnos que sí avanzan?” (párrs. 1597 - 1599). Pero una pedagogía inclusiva debe garantizar el acceso, la permanencia, la participación y el progreso de todos los estudiantes (Escobar et al., 2017).

Una profesora confiesa: “Confianza de expresar?, no, yo no, la verdad no” (párrs. 95-98). Cuando es necesaria la confianza para establecer relaciones afectivas que permitan que la práctica educativa se desarrolle desde la reflexión conjunta (Matus, 2013). El compromiso del docente por el trabajo se producirá solo cuando se ofrezcan vínculos de confianza que crecen y se fortalecen al manifestar aprecio hacia el otro (García, 2013). El director afirma que demuestra su confianza a los profesores de la siguiente forma:

En el trato; se puede ser director impositivo, autoritario, se puede ser despectivo, se crea confianzas de apoyo académico, profesional, docente y hasta humano. Entonces a ellos, al final, los compromete a hacer bien su trabajo, ¿no? Esa es la forma en que yo les demuestro que les ayudo: con materiales, con archivos, con exceles, con Word (párrs. 1656-1660).

En relación con la categoría Preferencias del director con el personal, la mayoría del profesorado reconoce que existen tratos diversos hacia los docentes. En el primero de los casos, una docente menciona que el director "dice bien feo las cosas, hasta uno se siente mal porque es muy cargado y a veces a unas maestras se los dice bonito y a otras no, como que tiene a sus favoritos” (párrs. 1047-1050). Posteriormente, la misma docente refiere: 


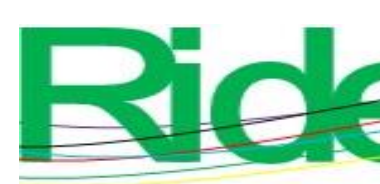

Revista Iberoamericana para la
Investigación y el Desarrollo Educativo
ISSN $2007-7467$

Hace tiempo le dio un permiso de 20 días a la maestra $\mathrm{F}$ por cosas de su esposo, que ponle, está bien porque es la salud ¿verdad?, pero no nos ha dado permisos económicos a los demás, y eso es algo que nos molesta y ya nos dijo que en noviembre tampoco va a dar (párrs. 1794-1799).

Maestros, alumnos y todos los miembros de la comunidad escolar deben generar un sentido de comunidad basado en la satisfacción en el trabajo, la motivación y la identidad hacia el centro. En la escuela primaria en cuestión, sin embargo, se evidencian situaciones como la siguiente:

El dire iba atrás de ella y le decía bien feo: “¡ya cállese, no me diga nada!” Y ahí estaba la pobre maestra llorando cuando se fue. A mí en una ocasión también me gritó enfrente de los otros profesores ahí en la Dirección y ninguno le dijo nada (párrs. 2505-2508).

(...) Yo sí le dije: “no, dire, no tiene por qué hablarme así, yo sí le hago lo que me dice cuando me dice, no tiene por qué tratarme así”. Y desde entonces no me ha vuelto a hablar así. Pero yo me defendí, la maestra E deja que le diga todo eso, enfrente de todos (párrs. 2218-2223).

Respecto a la categoría de Evidencia de falta de trabajo colaborativo, una maestra expresó que "aquí ya no se quieren meter en esos problemas de niños especiales. Cuando yo llegué aquí me sorprendí mucho de que no se les diera tanta atención a los niños" (párrs. 1965-1967). Confirmando esta expresión, otra de las docentes compartió una consulta que le realizó a una de sus compañeras:

Si podía canalizarle a otro niño a la maestra de educación especial para que ella me asesorara, pero dijo que ella estaba muy ocupada y solo le recibía a la niña que no habla, pero no se había podido llevar al niño (párrs. 213-215)

Esto a pesar de que una de las ventajas del trabajo en colaboración es la organización compartida del trabajo (Fernández y Hernández, 2013), el incremento del compromiso y responsabilidad por la educación de los alumnos y el desarrollo profesional del docente que le brinda identidad en el centro (Matus, 2013).

En el cumplimiento de la función que corresponde a cada miembro, un profesor menciona que: "el director no nos dijo nada o al menos no a todos, pero es porque a veces le dice a dos, tres y se le olvida decirle a los demás" (párrs. 436-438). Otra maestra refiere que "mi sentir aquí es que falta un poquito más de comunicación entre todos los maestros, como 


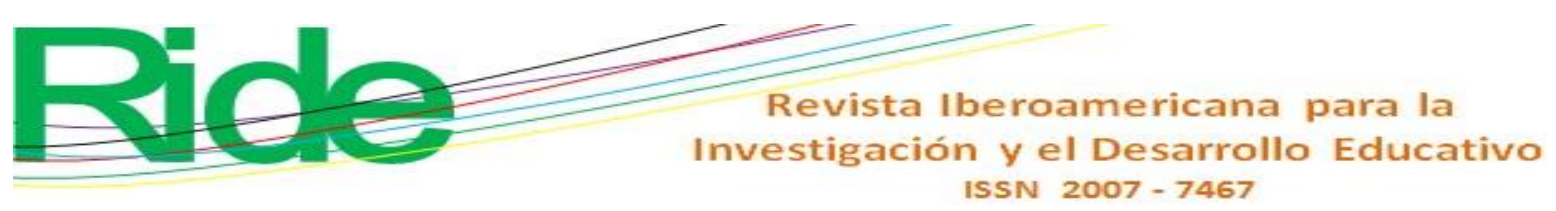

que, en cuanto a los maestros, sí nos falta un poquito más unirnos, este, de decir, compartir" (párrs. 157-158). Por otro lado, también los docentes afirman que "no hacemos actividades en comunidad, es muy difícil, lo único que se puede hacer son desfiles, pero es muy difícil, solamente como las de hoy con los padres y aun así ni vienen" (párrs. 719-721).

Una profesora recuerda: "Se formó una colaboración porque cada uno hizo lo que le tocaba del reparto del trabajo y se involucró a los padre de familia, que era en donde estábamos batallando" (párrs. 1724-1726). El director, a su vez, dice que las reuniones que el grupo docente realiza tienen el objetivo "de tomar acuerdos. Ustedes pueden expresar su molestia, hasta su ánimo en la escuela, pero también los demás" (párrs. 1698-1672). Sin embargo, una maestra afirma: "En los consejos técnicos a veces ni participo porque es muy grosero y empieza con sus albures, a veces hasta me salgo" (párrs. 1035-1045). Otra docente agrega que en estas reuniones "comparten sus dudas y problemas para que den sugerencias, pero nada más dicen que le eches ganas o fallas en esto, debes hacerle así, pero no para ayudarte, nomás para criticarte" (párrs. 1731-1735).

El líder escolar impone su percepción sobre el hecho de la participación de los padres de familia en las actividades de la institución. Al respecto, él considera lo puesto a continuación:

Lo primero es avisarles a los maestros que traten que su relación con el padre de familia sea meramente de trabajo. Ese es el primer principio, que uno debe, como trabajador, no intimar emocionalmente con los padres de familia. Mucho menos con las madres de familia, ¿verdad? Cumplo mi trabajo, ahí me voy. Hice mi trabajo, yo me voy. Porque esa es la ventaja que tenemos los maestros, que no vivimos en el lugar de nuestro trabajo, no hay ese acercamiento (párrs. 1204-1209). 


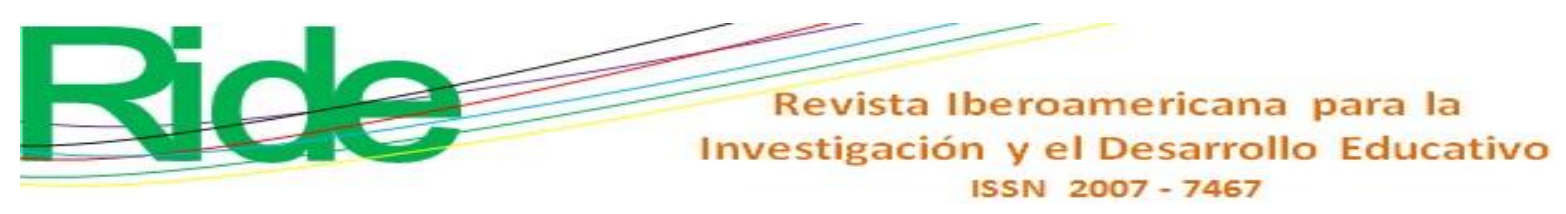

\section{Discusión}

La inclusión educativa es el resultado de un conjunto de variables que coinciden en un solo vértice: atención a la diversidad. Esta orientación de intervención en la escuela es una filosofía que implica la necesidad de sumar la labor colegiada del cuerpo docente y paradocente, así como estrechar una alianza de trabajo con los padres de familia que permita que el centro de la fórmula sea el alumno. No puede concebirse la inclusión educativa desde una variable; es necesaria la participación de cada elemento, tal vez unos en mayor medida que otros, pero no su ausencia total.

Difícilmente puede afirmarse que una escuela trabaja con la filosofía de inclusión educativa si no se observa que la toma de decisiones en el día a día se hace con principios democráticos y no mediante actitudes autoritarias. La inclusión educativa no es un conjunto de "buenas acciones" hacia los alumnos, implica que se viva en el plantel en un ambiente de libertad y reconocimiento de los derechos de todos los miembros de la comunidad escolar, así como respeto y camaradería. Es una filosofía que se adopta para lo cotidiano.

En la tabla 3 se puede apreciar que la inclusión educativa tiene dos dimensiones o categorías: cultura y políticas inclusivas. En esas categorías prevalece la intención de integrar equipos, hacer trabajo colaborativo, se reconoce a la persona y se da sentido de pertenencia, se erradican las prácticas de discriminación y se considera a todos los alumnos por igual, se estimula la solidaridad y se fomenta el respeto por la diversidad. Además, se establecen las políticas y lineamientos para la operación de la escuela con esa filosofía de trabajo. 
Tabla 3. Variables y dimensiones de la inclusión educativa

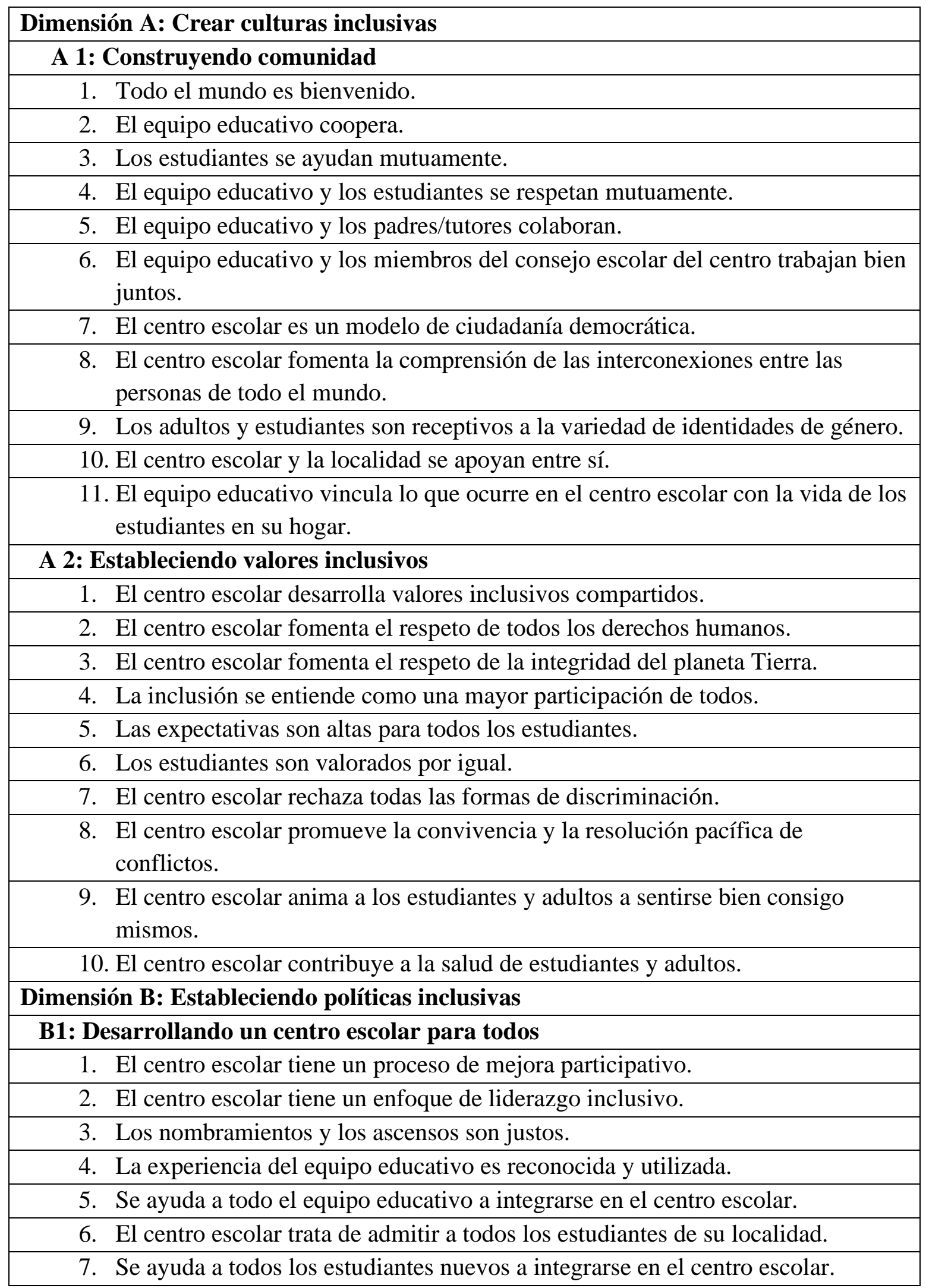




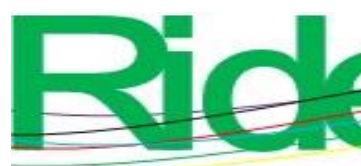

Revista Iberoamericana para la Investigación y el Desarrollo Educativo ISSN $2007-7467$

Desde la psicología educativa se puede planear un trabajo de intervención para ayudar a la comunidad escolar. Primero, a reconocer que es necesario que se transformen, que se muevan, que recuperen la emoción de trabajar y aprender con los niños. Es posible generar una conciencia de trabajo colectivo si se visualizan como capaces de hacerlo y si en el camino, durante el proceso de intervención, se puede incidir en la conciencia de los alumnos para que se motiven en temas de su interés. Entonces, la apuesta de trabajar con la filosofía de la educación inclusiva deja de ser "una feliz utopía". Puesto que, aunque nos propone metas lejanas, en el camino que se recorre por querer alcanzarlas, siempre salimos ganando, sin importar que se tenga que seguir intentándolo una y otra vez.

\section{Conclusiones}

Como consecuencia de su propia práctica, la escuela primaria no se desarrolla en los principios inclusivos, ni considera las necesidades de su contexto para la mejora de la calidad educativa. Bajo las condiciones que se han adoptado en la institución, no se permiten los encuentros prolongados entre los docentes, ni a los padres de familia con el profesor dentro del aula. Existen sospechas, prejuicios o malentendidos evidenciados a través de comentarios por parte del grupo directivo hacia el personal, pero sin una retroalimentación positiva de las tareas realizadas o la puesta en marcha de ideas y propuestas de innovación.

Los docentes tienen poca seguridad para participar en la toma de decisiones y en la generación de ideas al trabajar colaborativamente en reuniones. Los apoyos que reciben son críticas sobre su trabajo o consejos poco útiles para implementar, más que por el enriquecimiento personal y profesional o por solucionar las necesidades reales que existen en sus aulas. No son evidentes los vínculos afectivos entre compañeros; tampoco las ayudas ni apoyos. No existe la participación activa del padre de familia en el centro escolar.

La escuela primaria localizada en la periferia de la ciudad de Durango no es un centro escolar inclusivo que implemente en su práctica el trabajo colaborativo como estrategia para alcanzar una mejora educativa constante. Esta evidencia invita a considerar que es necesario una segunda etapa de análisis, en donde surja un programa que permita la creación de una escuela inclusiva que busque la participación e involucramiento de todos los miembros de la comunidad escolar. 


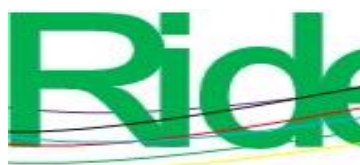

Revista Iberoamericana para la Investigación y el Desarrollo Educativo ISSN $2007-7467$

De lo anteriormente expresado se desprende la necesidad de que en la escuela se reconozca el problema para que se dé inicio un proceso de transformación. Con el diagnóstico elaborado se muestran evidencias de falta de trabajo colaborativo, de liderazgo autocrático y de falta de conocimiento integral sobre la inclusión educativa. Pero sobre todo debe considerarse que el trabajo del colectivo de docentes no está orientado a aplicar los principios de educación para todos y de inclusión educativa. Estos principios no son su objetivo de trabajo, ya que sigue expresándose el modelo médico de intervención del subsistema de educación especial; no es un centro educativo en donde se pregunten ¿qué debemos hacer para que todos aprendan? ellos se siguen preguntando o ¿qué tiene que hacer el niño para aprender? La primera pregunta es de filosofía inclusiva, de transformación del centro escolar, de eliminación de barreras para el aprendizaje. La segunda es para que no suceda nada en la escuela, pero, lamentablemente, muy poco orientada a que los alumnos sean considerados como personas diversas.

\section{Referencias}

Barrios, N., Castillo, M., Fajardo, F., Rojas, J. y Nova, A. (2004). El aula, un escenario para trabajar en equipo: Características de las acciones mediadas donde se favorecen las competencias laborales generales interpersonales. (tesis de maestría). Pontificia Universidad Javeriana, Bogotá.

Beltrán, Y., Martínez, Y. y Torrado, O. (2015). Creación de una comunidad de aprendizaje: una experiencia de educación inclusiva en Colombia. Revista Encuentros, 13(2), 5772. Recuperado de doi.org/10.15665/re.v13i2.498.

Bolívar, A. (2013). La lógica del compromiso del profesorado y la responsabilidad de la escuela: una nueva mirada. Revista Iberoamericana sobre Calidad, Eficacia y Cambio en Educación, 11(2), 60-83. Recuperado de http://www.redalyc.org/articulo.oa?id=55127024003.

Booth, T. y Ainscow, M. (2011). Guía para la Educación Inclusiva: Desarrollando el aprendizaje y la participación en los centros escolares. Madrid, España: FUHEMOrganización de Estados Iberoamericanos para la Educación, la Ciencia y la Cultura (OEI). Recuperado

de https://www.oei.es/historico/publicaciones/detalle_publicacion.php?id=151. 


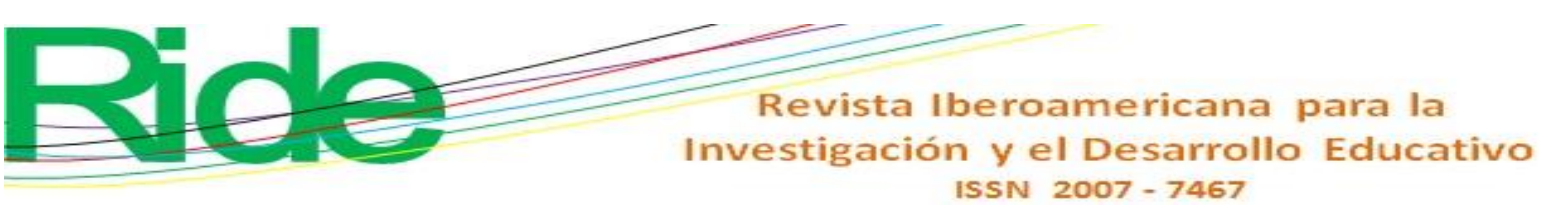

Echeita, G. (2013). Inclusión y exclusión educativa. "Voz y quebranto". Revista Electrónica Iberoamericana sobre Calidad, Eficacia y Cambio en Educación, 11(2), 9-18. Recuperado de http://www.redalyc.org/articulo.oa?id=55127024005.

Echeita, G. y Ainscow, M. (2011). La educación inclusiva como derecho. Marco de referencias y pautas de acción para el desarrollo de una revolución pendiente. Tejuelo, 12, 26-46. Recuperado de https://dialnet.unirioja.es/servlet/articulo?codigo=3736956.

Escobar, E., Albores, A. y Guillén, D (coords). (2017). Guía para una escuela inclusiva. Chiapas, México: Cresur.

Flores, V., García, I. y Romero, S. (2017). Prácticas inclusivas en la formación docente en México. Liberabit, 23(1), 39-56. Recuperado de https://doi.org/10.24265/liberabit.2017.v23n1.03.

García, E., García, A. y Reyes, J. (2014). Relación maestro alumno y sus implicaciones en el aprendizaje. $R a$ Ximhai, 10(5), 279-290. Recuperado de http://www.redalyc.org/articulo.oa?id=46132134019.

García, K. (2013). El aprecio: fortalece al individuo y la organización. San Juan, Puerto Rico: Oficina de Etica Gubernamental de Puerto Rico.

Granada, M., Pomés, M. y Sanhueza, S. (2013). Actitud de los profesores hacia la inclusión educativa. Papeles de trabajo, (25), 51-59. Recuperado de http://www.scielo.org.ar/pdf/paptra/n25/n25a03.pdf.

Gutiérrez, M., Martín, M. y Jenaro, C. (2014). El Index para la inclusión: presencia, aprendizaje y participación. Revista nacional e internacional de educación inclusiva, 7(3), 186-201. Recuperado de https://www.researchgate.net/publication/277141157.

López, M. (2011). Barreras que impiden la escuela inclusiva y algunas estrategias para construir una escuela sin exclusiones. Innovación educativa, (21), 37-54. Recuperado de http://www.usc.es/revistas/index.php/ie/article/download/23/140.

Matus, L. (2013). La construcción de una identidad docente, ¿un desafío para la política educativa? $\quad$ Exitus, 3(1), 75-87. Recuperado de http://www.ufopa.edu.br/portaldeperiodicos/index.php/revistaexitus/article/view/25 0 .

Moliner, O. (2013). Educación inclusiva. España: Edición de la Universitat Jaume I. 


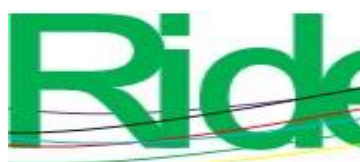

Revista Iberoamericana para la Investigación y el Desarrollo Educativo ISSN $2007-7467$

Muntaner, J., Rosselló, M. y De la Iglesia, G. (2016). Buenas prácticas en educación inclusiva. Educatio Siglo XXI, 34(1), 31-50. Recuperado de doi.org/10.6018/j/252521.

Organización de las Naciones Unidas para la Educación, la Ciencia y la Cultura [Unesco]. (2004). Temario abierto sobre educación inclusiva. Santiago, Chile: Editorial Organización de las Naciones Unidas para la Educación, la Ciencia y la Cultura. Recuperado de http://sid.usal.es/idocs/F8/FDO23200/temario_abierto_educacion_inclusiva.pdf.

Organización de las Naciones Unidas para la Educación, la Ciencia y la Cultura [Unesco]. (2004). Temario abierto sobre educación inclusiva. Santiago, Chile: Editorial Organización de las Naciones Unidas para la Educación, la Ciencia y la Cultura. Recuperado de http://sid.usal.es/idocs/F8/FDO23200/temario_abierto_educacion_inclusiva.pdf.

Porter, G. y Towell, D. (2017). Promoviendo la educación inclusiva: claves para el cambio transformacional en los sistemas de educación. (tríptico). Reino Unido: Inclusive Education Canada \& Centre for Inclusive Futures.

Sanabria, A. y Castañeda, M. (2016). Trabajo en equipo o equipo de trabajo: ¿es posible en el sistema de salud colombiano? Revista Colombiana de Cirugía, 31(2), 98-102. Recuperado de http://www.redalyc.org/articulo.oa?id=355545923004.

San Martín, C., Villalobos, C., Muñoz, C. y Wyman, I. (2017). Formación inicial docente para la educación inclusiva: análisis de tres programas chilenos de pedagogía en educación básica que incorporan la perspectiva de la educación inclusiva. Revista Calidad en la educación, (46), 20-52. Recuperado de https://scielo.conicyt.cl/pdf/caledu/n46/0718-4565-caledu-46-00020.pdf.

Sisto, V. (2009). Cambios en el trabajo, identidad e inclusión social en Chile: desafíos para la investigación. UNIVERSUM, 3(24), 192-216. Recuperado de https://scielo.conicyt.cl/pdf/universum/v24n2/art_11.pdf. 


\section{Revista Iberoamericana para la Investigación y el Desarrollo Educativo \\ ISSN 2007 - 7467}

\begin{tabular}{|c|c|}
\hline Rol de Contribución & Autor (es) \\
\hline Conceptualización & Fernanda Bonilla Serrato. Igual Jaime Fernández Escárzaga. Igual \\
\hline Metodología & Jaime Fernández Escárzaga. Principal Marco Antonio Vázquez Soto. Que apoya \\
\hline Software & NO APLICA \\
\hline Validación & $\begin{array}{l}\text { Fernanda Bonilla Serrato. Igual Jaime Fernández Escárzaga. Igual } \\
\text { Marco Antonio Vázquez Soto. Que apoya }\end{array}$ \\
\hline Análisis Formal & $\begin{array}{l}\text { Fernanda Bonilla Serrato. Igual Jaime Fernández Escárzaga. Igual } \\
\text { Marco Antonio Vázquez Soto. Que apoya }\end{array}$ \\
\hline Investigación & Fernanda Bonilla Serrato. Principal Jaime Fernández Escárzaga. Que apoya \\
\hline Recursos & Jaime Fernández Escárzaga \\
\hline Curación de datos & Jaime Fernández Escárzaga. Principal Marco Antonio Vázquez Soto. Que apoya \\
\hline $\begin{array}{l}\text { Escritura - Preparación del borrador } \\
\text { original }\end{array}$ & Fernanda Bonilla Serrato \\
\hline Escritura - Revisión y edición & Jaime Fernández Escárzaga. Igual Marco Antonio Vázquez Soto. Igual \\
\hline Visualización & $\begin{array}{l}\text { Fernanda Bonilla Serrato. Igual Jaime Fernández Escárzaga. Igual } \\
\text { Marco Antonio Vázquez Soto. Que apoya }\end{array}$ \\
\hline Supervisión & Jaime Fernández Escárzaga \\
\hline Administración de Proyectos & Jaime Fernández Escárzaga \\
\hline Adquisición de fondos & Jaime Fernández Escárzaga \\
\hline
\end{tabular}

